Cahiers de recherches médiévales

Journal of medieval studies

4 | 1997

Être père à la fin du Moyen Âge

\title{
Pour retrouver un père...La promotion du culte de saint Joseph au temps de Gerson
}

Paul Payan

\section{(2) OpenEdition \\ 12 Journals}

Édition électronique

URL : https://journals.openedition.org/crm/959

DOI : $10.4000 / \mathrm{crm} .959$

ISSN : 1955-2424

Éditeur

Honoré Champion

Édition imprimée

Date de publication : 15 décembre 1997

ISSN : $1272-9752$

\section{Référence électronique}

Paul Payan, « Pour retrouver un père...La promotion du culte de saint Joseph au temps de Gerson », Cahiers de recherches médiévales [En ligne], 4 | 1997, mis en ligne le 15 janvier 2007, consulté le 15 décembre 2022. URL : http://journals.openedition.org/crm/959; DOI : https://doi.org/10.4000/crm. 959

Ce document a été généré automatiquement le 15 décembre 2022.

Tous droits réservés 


\section{Pour retrouver un père...La promotion du culte de saint Joseph au temps de Gerson ${ }^{1}$}

Paul Payan

1 C'est une scène de famille toute simple: un homme et une femme qui entourent leur petit enfant. Le personnage principal, c'est cet enfant, et juste à côté, sa mère. Le troisième, en retrait, se fait presque oublier, et pourtant c'est lui qui donne à la scène un lien avec la société humaine en brisant l'intimité du couple mère-enfant. Cette image, tout l'Occident médiéval la connaît et l'identifie comme étant celle de la Nativité du Christ, la représentation du mystère de l'Incarnation, le fondement de la théologie chrétienne. Avec l'Annonciation et la Crucifixion, c'est l'une des images les plus diffusées dans le monde chrétien.

2 La réflexion sur la paternité dans la société du Moyen Âge doit donc nécessairement se confronter à cette image du père terrestre $d u$ Christ, saint Joseph. Par sa singulière position, ce personnage pourrait être une clé pour comprendre la conception chrétienne de la paternité, au cœur du mystère de l'Incarnation : voilà un homme qui se comporte comme un père, qui est considéré comme un père, mais qui n'a aucun lien naturel avec son enfant ${ }^{2}$. De plus, ce curieux père se prosterne devant ce fils et s'occupe humblement des tâches ménagères. Dans une société médiévale habituellement présentée comme patriarcale, une telle image suscite l'interrogation,... ou du moins elle le devrait.

3 On est frappé en effet de constater que saint Joseph est pour l'instant une zone d'ombre de l'historiographie, tout au moins française ${ }^{3}$. Il semble définitivement acquis que Joseph est un vieillard ridicule, un mari trompé et content de l'être, cible de moqueries irrévérencieuses, en contrepoint de la dévotion à la Vierge et à l'Enfant. C'est une opinion constamment reprise depuis sa formulation par Huizinga : «L'intérêt indiscret porté au père nourricier est pour ainsi dire la contrepartie de l'amour et de l'exaltation de Marie. Au fur et à mesure que s'élevait la figure de la Vierge, celle de Joseph tournait 
à la caricature. Les arts plastiques lui donnaient un type qui approchait de celui du paysan ridicule $»^{4}$.

On retrouve par exemple cette idée chez Louis Réau ${ }^{5}$, et plus récemment encore chez Jean Wirth qui évoque une «ridiculisation de l'institution fondamentale de la parenté humaine - le mariage - en la personne de saint Joseph, du travailleur marié qui gagne de ses mains la subsistance du ménage. Autour de 1400, saint Joseph est devenu le souffre-douleur des artistes et aucun saint n'a été si régulièrement maltraité $»^{6}$. Dernièrement, un article de Louise 0 . Vasvari sur la célèbre image de Joseph dans son atelier du Retable de Mérode insistait sur l'aspect caricatural du personnage, le type même du mari trompé des fabliaux : attentif à son travail de menuisier, il ignore que la Vierge est en train d'accueillir l'Ange de l'Annonciation'.

5 Il n'est pas question de remettre en cause le bien fondé de ces analyses qui reposent sur quelques images célèbres où Joseph est clairement ridiculisé. Jean Gerson lui-même se plaignait que Joseph soit représenté sous la forme d'un vieillard, malgré quelques exemples où il apparaît in aetate juvenili et que le chancelier aurait rencontré in Almania. Mais il ne consacre que quelques mots à cette constatation, et évacue le problème en citant la fameuse formule d'Horace: Pictoribus atque poetis / Quidlibet audendi semper fuit aequa potestas 8 .

6 Il faut reconnaître que les exemples d'un Joseph ridiculisé sont assez faciles à trouver parmi les retables ou les enluminures du début du $\mathrm{XV}^{\mathrm{e}}$ siècle. Il est vieux, fatigué voire endormi, il se chauffe les pieds ou s'occupe de la soupe, se désaltère pendant la Fuite en Égypte. Mais encore faut-il rester prudent, et ne pas confondre modestie et ridicule, effacement et mise à l'écart. Joseph peut aussi avoir une attitude contemplative, soit en retrait soit sur le même plan que la Vierge ; s'il s'occupe de tâches matérielles, cela peut être une façon de mettre en valeur son rôle de père nourricier et protecteur. On comprend que ce problème soit délicat car il met en œuvre un concept très subjectif et dépendant étroitement du contexte culturel. Afin de nous faire une idée de la question, nous avons examiné un corpus-test d'environ 300 images de Joseph provenant plus la plupart de l'enluminure française des années 1370-1450. Or, les cas où Joseph est incontestablement ridicule sont minoritaires - à peine $10 \%$ de notre corpus. On les rencontre essentiellement dans l'art courtois des années 1400-1420. Par la suite, le thème du Joseph contemplatif devient dominant et conduit aux représentations de la Sainte Famille du XVI ${ }^{\mathrm{e}}$ siècle. Bien entendu, ceci n'est que le premier résultat d'une recherche en cours, une hypothèse de travail en quelque sorte, mais qui nous incite à aller au-delà de l'idée d'un Joseph ridiculisé et mis à l'écart.

7 Sa position, par son humilité même, lui confère un rôle essentiel dans l'image : il est, dans la plupart des cas, un spectateur modeste de la scène, et un miroir d'un ou plusieurs personnages ${ }^{9}$. Par conséquent, celui qui regarde l'image peut facilement s'identifier à lui, modeste reflet de Dieu le Père, des rois ou des prêtres, et donc imago au sens médiéval du terme ${ }^{10}$.

8 Pour saisir toute l'ambiguïté de notre personnage, il faut donc le replacer dans un contexte iconographique, théologique et spirituel beaucoup plus vaste, en concentrant l'enquête sur le tournant des $\mathrm{XIV}^{\mathrm{e}}$ et $\mathrm{XV}^{\mathrm{e}}$ siècles, période qui nous semble fondamentale pour ce sujet. C'est l'époque des grands enlumineurs parisiens chez qui l'on rencontre les images les plus célèbres d'un Joseph ridiculisé et mis à l'écart, mais aussi les premières représentations d'une adoration de l'Enfant où le père prend une place aussi importante que la Vierge ${ }^{11}$. C'est aussi l'époque des premiers grands Mystères, anglais 
et français, dans lesquels notre personnage tient un rôle non négligeable ${ }^{12}$. C'est surtout le moment où prend forme la dévotion à saint Joseph et où s'affine la réflexion théologique à son égard, notamment autour du chancelier Jean Gerson. Enfin, c'est une période clé pour une réflexion plus générale sur la paternité, à un moment de crise de l'autorité qu'elle soit religieuse ou politique, et de bouleversement des cellules familiales, après les grandes épidémies.

Tous les historiens de la dévotion à saint Joseph s'accordent pour considérer l'action du chancelier de l'Université de Paris Jean Gerson en sa faveur comme un moment essentiel dans l'instauration de son culte. Nous partirons donc ici de ces grands textes pour faire le point sur la place de l'époux de la Vierge dans la théologie et la dévotion du début du $\mathrm{XV}^{\mathrm{e}}$ siècle.

Au mois d'août 1413, Gerson rédige une Épitre sur le culte de saint Joseph qu'il adresse «à toutes les églises, spécialement à celles qui sont dédiées à Notre Dame ». Il leur demande instamment d'instaurer une fête pour commémorer le mariage de la Vierge et de son époux terrestre ${ }^{13}$. C'est la première intervention du chancelier de l'Université de Paris en faveur de saint Joseph. C'est aussi le premier d'une longue série de textes, pour la plupart écrits entre 1413 et 1416 et dans lesquels Gerson développe sa réflexion théologique et pastorale sur le père terrestre du Christ, tout en se battant pour la promotion de son culte. Un mois après son premier appel, le 26 septembre 1413, il réitère sa demande d'une fête en l'honneur du mariage de la Vierge ${ }^{14}$. Le 23 novembre, il écrit au duc de Berry pour l'exhorter à instituer une fête en commémoration du mariage de Joseph et Marie dans l'église Notre-Dame ${ }^{15}$. C'est sûrement à la fin de cette même année 1413 qu'il rédige les Considérations sur saint Joseph, un long texte en français où il précise les raisons qui justifient une promotion de son culte ${ }^{16}$. Entre 1414 et 1416, selon la chronologie établie par Max Lieberman, il compose un long poème latin, la Josephina. Enfin, le 8 septembre 1416, jour de la fête de la Nativité de la Vierge, il s'adresse aux pères du concile de Constance pour un long sermon sur l'Évangile du jour, Jacob autem genuit Joseph... (Matth. I, 16). Cette magistrale démonstration, plus scolastique qu'oratoire comme il l'avoue lui-même ${ }^{17}$, est l'aboutissement de sa réflexion sur le sujet. Autour de ces grands textes s'articule toute une série de poèmes, d'oraisons, et de multiples allusions dans des sermons ou des lettres ${ }^{18}$.

11 La chronologie de ces textes, à elle seule, suscite l'interrogation : pour quelles raisons Gerson s'est-il subitement lancé, entre 1413 et 1416, dans cette action en faveur d'un personnage en apparence si effacé et secondaire ? Certes, celui-ci correspond bien à la sensibilité spirituelle du chancelier. Il pouvait trouver dans ce personnage humble et pourtant si proche du mystère de l'Incarnation un modèle pour sa dévotion personnelle, moderne, un exemple de cette spiritualité de méditation intime, de contemplation secrète qu'il a si bien diffusée dans sa pastorale. Il y retrouvait aussi certainement un souvenir de ses origines modestes. Dans les Considérations sur saint Joseph, il s'exclame: «la benignité et l'humanité de Dieu fu tele qu'il ha voulu estre subget a ung fevre en bois, c'est a dire a ung charlier ou charon, ou a ung charpentier ${ }^{19}$. Or, on se souvient que Gerson s'appelle Jean Charlier! Le long poème de la Josephina commence d'ailleurs par une description du pèlerin, que l'on a très vite interprétée comme un autoportrait: les rares représentations de Gerson dans l'iconographie du $\mathrm{XV}^{\mathrm{e}}$ siècle le montrent ainsi ${ }^{20}$. Mais si l'on comprend assez facilement que la dévotion de Gerson se porte sur le père terrestre du Christ, pourquoi faut-il attendre 1413 pour le voir intervenir en sa faveur? 
12 Cette année-là fut particulièrement difficile pour le chancelier de l'Université de Paris, qui s'était attiré les foudres des Bourguignons pour ne pas avoir apprécié la théorie de Jean Petit en faveur du tyrannicide. Au mois de juillet, Gerson voit sa maison pillée par les Cabochiens, et il doit s'enfermer dans Notre-Dame. C'est donc juste après la paix de Pontoise du 28 juillet qu'il éprouve le besoin de lancer son premier appel en faveur de l'instauration d'une fête pour le mariage de la Vierge. En même temps, il s'attaque à la Justification du duc de Bourgogne et à l'éloge du tyrannicide de Jean Petit dans un long discours prononcé devant le roi et plusieurs grands princes : Rex in sempiternum vive. Ce texte se termine par la joie de la paix retrouvée,

grace à Dieu de amour et de paix, qui est vie, voye et verite (Jean XIV, 6). Grace à sa glorieuse Vierge Mere, et à sainte Geneviesve, qui oncques ne faillirent à ceste cité. Grace au virginal espoux de Notre Dame, saint Joseph juste, du quel mariage fut signification de la plus parfaicte union et coniunction qui soit : c'est de Dieu et de son Église". Si devons honourer ce mariage virginal, ceste sacrée et très caste coniunction, nous qui querons paix et union"

13 On voit alors fort bien quelle est la fonction de Joseph dans cette conjoncture difficile, face à des pouvoirs divisés, aussi bien à Paris qu'à Constance. Pour Gerson, l'époux de la Vierge est avant tout un modèle d'union dans la paix, et dans les circonstances des années 1410, cette image apparaît comme une criante nécessité. C'est sur cette idée qu'il conclut son grand sermon de Constance, le 8 septembre 1416 : l'intercession de Joseph, dit-il, rendra à l'Église « un homme unique, vrai et juste, le Souverain Pontife, son époux à la place du Christ $»^{23}$.

14 La démarche de Gerson en faveur de l'époux de la Vierge est donc totalement liée aux conflits politiques du moment : le mariage de Joseph et de Marie est un modèle de paix et d'union face aux divisions de l'Église et des nations. Mais ce n'est pas la seule portée politique que Gerson tire de sa réflexion sur l'époux de la Vierge. Dans les Considérations sur saint Joseph, puis dans son discours du 8 septembre 1416, il se fait l'écho d'un débat théologique qui nous ramène au cœur du conflit franco-anglais : l'origine de la royauté terrestre du Christ.

Si dient aucuns docteurs que le royaume des Juifs appartennoit a Nostre Dame par prochaine succession et heritage, a cause de Joachin son pere, et de sainte Anne sa mere; et a ceste cause dient que Jhesucrist fut vray roy des Juifs par droit a cause de sa mere Nostre Dame; laquelle chose ont voulu les Anglois alleguer aucune fois pour eulz, en disant que femme povoit bien succeder au royaume de France, selond la response de Dieu en l'ancien testament des filles".

15 Une telle théorie justifierait en effet les prétentions des rois d'Angleterre à la couronne de France, depuis la mort de Charles IV le Bel, en 1328. Dans son traité, Gerson ne prend pas la peine de discuter cet argument: "si laisse ceste matiere a la disputacion des clercs ». Mais la construction même de son texte lui permet de désamorcer le problème avant même de l'avoir posé : dans le paragraphe précédent, il venait de rappeler la descendance royale de Joseph.

Ceste noblesse royale fu telle en David et si prochaine que selond aucuns docteurs le royaume des Juifs appartenoit a Joseph par legitime succession, et de ce dient que Jhesucrist pour ceste cause fu appele roy des Juifs plus que des Romains ou des Français, comme celui qui estoit filz de Joseph, non mie par generation charnelle mais comme est dessus exposé, par le droit qu'il avoit au corps Nostre Dame ${ }^{3 .}$.

16 Ainsi, grâce à Joseph, l'héritage paternel existe bien, et permet de répondre implicitement à l'argument anglais ${ }^{26}$. 

l'existence de ce débat, inter duo regna christianorum clarissima, Franciae et Angliae ${ }^{27}$. Mais, dans un passage qui, semble-t-il, n'a pas été prononcé, il donne cette fois son opinion en ramenant le débat sur la royauté du Christ à un niveau spirituel: «Je crois plus probable que le règne du Christ sur la Judée n'était pas temporel, mais spirituel, ainsi que pour les autres pays ${ }^{28}$ ». Malgré tant de prudence, Gerson maniait là des idées très dangereuses, touchant un problème alors ultrasensible. Il s'était déjà fait des ennemis dans le clan bourguignon en attaquant la doctrine de Jean Petit. En 1415, Martin Porée, évêque d'Arras et principal ambassadeur de Jean sans Peur, l'avait ouvertement attaqué en plein concile. Or, on possède un manuscrit rédigé pour Martin Porée sur l'affaire Jean Petit $^{29}$. On y lit deux notes sur le sermon de Gerson, la première sans date, la seconde reçue par l'évêque en avril 1417. Gerson y est accusé de justifier la succession par les femmes afin de s'attirer la faveur des Anglais ${ }^{30}$.

Gerson avait dit à peu près l'inverse, et ne cherchait en aucun cas à se rapprocher des Anglais! Ce curieux texte était sûrement destiné à calomnier Gerson, mais il pouvait aussi servir d'argument au moment même où Jean sans Peur reconnaissait la légitimité des prétentions du roi d'Angleterre.

Quoi qu'il en soit, il semble que Gerson ait eu trop d'ennemis, et que la question de Joseph ait été mal comprise ou trop controversée par les Pères du concile. Le 2 février 1418, le jour de la Purification de la Vierge, le chancelier prononce un nouveau sermon, Suscepimus, où il déplore que rien d'officiel n'ait été fait pour commémorer le mariage de la Vierge, alors que l'Église a retrouvé son unité ${ }^{31}$.

20 Sous la plume de Gerson, saint Joseph prend donc une importance inattendue : modèle de paix et d'union, il en devient aussi un modèle de vie à l'intention des grands de ce monde, nobles ou ecclésiastiques. Pour les premiers, il représente une noblesse vécue dans l'humilité du service; pour les seconds, il incarne l'équilibre entre la vie active et la vie contemplative. Cela permet à Gerson de lancer de violentes diatribes contre les mystiques qui «veullent (...) estre ravis iusques au tiers ciel sans avoir vesqu en discipline de la supplantacion des vices et mortification de leur charnalité ", puis contre les Mendiants « qui au moins se porroient occuper a honneste et paisible labeur, comme a escrire ou a corrigier livres, ou a aucun mestier qui ne requiert mie grande force ou distraction $»^{32}$.

21 On reconnaît bien ici la pastorale de Gerson, soucieuse d'équilibre dans la vie spirituelle, en réaction aux excès des mystiques ou de certains ordres mendiants. Le personnage de Joseph, humble dans son attitude mais noble par ses origines et son rôle, tour à tour serviteur et contemplatif, lui fournit un modèle tout indiqué, à l'intention de l'aristocratie politique et religieuse. Cela est déjà à souligner : le discours de Gerson concernant notre personnage s'adresse en priorité à ce public de choix, impliqué dans les grands débats de son temps, et non à un public populaire, dans le but d'une pastorale familiale pourtant chère au chancelier.

22 Cela peut s'expliquer par la place ambiguë de Joseph, père légal, père nourricier et protecteur, mais sans lien naturel avec son fils, et en situation d'infériorité par rapport à lui et à son épouse. Sa paternité est "putative», c'est-à-dire apparente, destinée à intégrer la Sainte Famille dans la société humaine, en camouflant en quelque sorte le mystère de l'Incarnation aux yeux des hommes ${ }^{33}$. Mais ce curieux statut confère à Joseph une importance fondamentale dans la définition d'une conception chrétienne de 
la paternité, au croisement entre deux traditions, la juive fondée sur les liens du sang, et la romaine, institutionnelle, permettant l'adoption. Contrairement à ce qu'on a pu dire, Joseph est donc très présent dans la réflexion théologique médiévale. On le rencontre principalement dans les écrits des premiers pères, en réaction aux hérésies trinitaires, puis aux XII ${ }^{e}$ et $\mathrm{XIII}^{\mathrm{e}}$ siècles, en liaison avec la réflexion sur le mariage. Dans le premier cas, il s'agit d'affirmer la réalité de la conception virginale du Christ, tout en justifiant le titre de père donné à Joseph. On trouve notamment chez Origène une double légitimation de la paternité de Joseph dans laquelle on peut retrouver les deux traditions, juive et romaine :

Pour quelle raison a-t-il appelé père celui qui ne l'était pas? Si l'on se contente d'une explication simple, on peut dire: le Saint-Esprit l'a honoré du titre de père parce qu'il avait nourri le Sauveur. Mais si l'on cherche quelque chose de plus élevé, on peut penser: la généalogie de Jésus se conduit de David à Joseph, et pour que Joseph ne semble pas nommé en vain, celui qui n'était pas vraiment le père du Sauveur reçut cependant ce titre pour donner lieu à la généalogie". 
Mais, désirant passer de la théologie à la dévotion, il cherche à leur donner un aspect plus concret, plus crédible, en reportant sur Joseph, à un moindre niveau, les grâces attribuées à Marie. Maîtrisant aussi bien le droit que la théologie, il combine habilement les différentes traditions juridiques et théologiques dans de subtils raisonnements destinés à l'auditoire du concile de Constance.

Le chancelier affirme tout d'abord clairement la virginité de Joseph, en réaction à une tradition populaire issue des apocryphes, selon laquelle celui-ci aurait été marié une première fois et aurait eu des enfants. Refusant cette hypothèse, Gerson en conclut que Joseph devait être relativement jeune au moment de son mariage avec la Vierge, entre trente et cinquante ans, tout au plus. Il fait pour cela appel au bon sens : un Joseph trop âgé et impotent aurait été la proie des moqueurs, ce qui aurait bouleversé les plans divins ${ }^{40}$. De plus, un mari trop vieux et faible serait un piètre secours pour la Vierge et son Enfant!

Mais Gerson ne s'arrête pas à ces remarques de bon sens. Poursuivant la logique d'une véritable union entre les deux époux, il en conclut que la Vierge devait être soumise à Joseph, «comme autre femme doit et peut faire a son espous. Vees doncques quele dignité c'est icy de Joseph que il soit chief et seigneur de la mere du chief et seigneur de tout le monde $»^{41}$. Plus encore, les deux époux furent unis, non seulement en esprit, en volonté et en biens, «mais furent une char et ung corps par union sacramentele, jasoice que point n'y fut honteuse commixtion $»^{42}$. Cette remarque permet à Gerson d'avancer avec prudence une hypothèse extrêmement originale sur la nature de la paternité de Joseph : puisque celui-ci et la Vierge ne forment qu'une seule chair, on peut donc dire que le Christ est né de la chair de Joseph et de Marie!

Le corps de Marie fut la propriété de Joseph par droit de mariage, qui réalise le transfert mutuel du corps de l'époux vis-à-vis de l'épouse, et inversement. Examinons si, en toute prudence, il nous serait permis de dire que le Christ Jésus est né du corps et de la chair de Joseph. On pourrait peut-être l'affirmer, si l'on ne craignait pas d'offenser les âmes pies".

Et qu'importe si l'auditoire reste sceptique : une incursion dans l'Ancien Testament lui permet de justifier la paternité de Joseph en appliquant la loi du lévirat ; le Saint-Esprit aurait fécondé la Vierge comme «frère » de Joseph.

Joseph fut donc père de Jésus par réputation, et aussi par les soins, parce qu'il nourrissait l'Enfant. Troisièmement, il fut père par génération, non pas certes par la sienne propre, mais par celle de Marie son épouse avec la coopération du SaintEsprit qui tint en quelque sorte la place de Joseph au moyen d'un souffle mystérieux plutôt que par une semence charnelle. Voilà pourquoi saint Joseph peut être considéré comme le père de l'Enfant Jésus, non selon la nature, mais selon la loi, lui à qui l'Esprit Saint a suscité une descendance plus puissante que celle d'un principe charnel".

31 Malgré la subtilité du raisonnement, celui-ci reste jusque-là assez traditionnel : il s'agit d'affirmer la réalité de la paternité de Joseph. Mais dans son désir de faire de Joseph un grand saint, objet de respect et de dévotion, Gerson se lance dans une théologie beaucoup plus audacieuse. On sait que le chancelier s'était déjà battu en faveur de l'Immaculée Conception dont l'idée ne faisait pas encore l'unanimité. Or, il propose ici pour Joseph une formule encore plus subtile : la sanctification in utero.

De même que Marie, de qui est né Jésus, a été sanctifiée dans le sein maternel avant sa naissance, ainsi peut-on le croire pieusement de Joseph, son virginal époux, mais non pas exactement de la même manière. On peut, semble-t-il, noter cette 
différence : Joseph a été sanctifié dans le sein de sa mère par le baptême de désir après avoir contracté le péché originel, comme saint Jean Baptiste et plusieurs autres". Sienne, puis par Bernardin de Bustis, un franciscain de la fin du XV $\mathrm{XV}^{\mathrm{e}}$ siècle ${ }^{53}$. Par la suite, elle ne sera évoquée que pour la réfuter ${ }^{54}$. 

ses inspirateurs, «feu Pierre Bourguignon Celestin au quel, comme il souloit dire, saint Joseph estoit especial patron et refuge ». Il s'agit selon M. Lieberman de Pierre Pocquet, né vers 1340 et entré au couvent des Célestins de Paris en 1369. Il est l'auteur, entre autres, d'un Dictamen de laudibus beati Joseph, une méditation émouvante sur la vie de la Sainte Famille, nourrie d'une solide théologie, parfois audacieuse. La théorie de la sanctification in utero n'est pas évoquée, mais le texte se termine par la description d'un Joseph glorifié au Ciel, et l'on sait que l'idée d'Assomption corporelle est liée à la notion de sanctification avant la naissance. De plus, on trouve dans ce texte une volonté moralisatrice assez forte, bien en rapport avec la spiritualité des Célestins, mais qui annonce aussi les sermons de Gerson ${ }^{62}$.

41 Il faut évoquer aussi un texte célèbre, le De duodecim honoribus sancti Josephi, du cardinal Pierre d'Ailly, le maître de Gerson, et son prédécesseur à la chancellerie de l'Université de Paris $^{63}$. Malheureusement, la datation de ce texte est incertaine, et son contenu, assez traditionnel, le démarque un peu de la pensée gersonienne. Il est tout de même intéressant de relever une fois de plus la présence de saint Joseph dans la réflexion d'une grande autorité ecclésiastique, très proche des problèmes du Grand Schisme. 
La dévotion à saint Joseph existe donc déjà au moment où Gerson se manifeste en sa faveur, mais de façon ponctuelle, à l'échelle d'un ordre mendiant, d'un individu ou d'une localité. On signale par exemple une église de Bologne consacrée à saint Joseph dès le XII ${ }^{\mathrm{e}}$ siècle ${ }^{64}$. Il s'agissait d'un petit oratoire appartenant à l'abbaye bénédictine de Sainte-Hélène. Or, cette abbaye est abandonnée et transférée aux Servites de Marie en 1299 , et saint Joseph devient alors le titre principal du monastère. Dans cette même ville de Bologne, on signale les premières utilisations de Joseph comme nom de baptême, et celui-ci rejoint la Litanie des Saints dès $1359^{65}$. Partout ailleurs, le prénom de Joseph n'apparaît pas avant le XVI ${ }^{\mathrm{e}}$ siècle ${ }^{66}$.

Ce mouvement, dont nous venons d'esquisser les grandes lignes, est donc finalement limité. Les sources que nous avons évoquées jusqu'à présent émanent toutes d'autorités ecclésiastiques, à l'intention d'un public restreint et choisi. Par exemple, Bernardin de Sienne évoque saint Joseph dans un sermon latin, et non dans ses Prediche volgari ${ }^{67}$. Gerson utilise Joseph dans sa réflexion écclésiologique et politique, et non dans sa pastorale familiale.

4 Il serait donc nécessaire de confronter ces grands textes théologiques et spirituels avec d'autres types de source qui nous permettraient peut-être d'atteindre une autre image de Joseph, celle qui est perçue par une plus large part du peuple chrétien, celle qui est véhiculée par les Mystères et l'iconographie. Les premières études, portant notamment sur les Mystères anglais, ont montré un Joseph profondément humain, et donc accessible à tous, en proie au doute, à la suspicion, au repentir, à l'émotion, à l'adoration ${ }^{68}$. Son rôle purement dramatique est donc fondamental dans l'apparition d'un théâtre moderne, alors que sa place dans l'iconographie nourrit une relecture de l'intimité familiale et de son rapport avec le sacré.

On le voit, la réflexion sur saint Joseph peut conduire assez loin, et dépasse largement la simple ridiculisation d'un vieux mari trompé. La figure de ce vieillard modeste apparaît lourde de sens. A travers elle, la question de la paternité apparaît sous un jour nouveau. On a vu que Gerson cherchait à présenter Joseph comme un modèle de noblesse vertueuse, d'union et de paix, d'équilibre entre vie active et contemplative, et ces vertus semblent surpasser la simple relation qu'il entretient avec son Fils. Mais celles-ci donnent peut-être à la paternité son véritable sens, celui d'une médiation entre le cercle familial et le monde social, entre le visible et l'invisible, l'humain et le divin $^{69}$. Or, la société du début du $\mathrm{XV}^{\mathrm{e}}$ siècle semble avoir un besoin aigu d'une telle médiation, face aux déchirures de l'Église, à la crise de l'autorité politique, aux bouleversements des structures familiales. A travers Joseph et quelques autres figures de pères, on assiste en quelque sorte à la recherche d'une nouvelle conception - ou d'une redéfinition - de la paternité. Celle-ci met en cause les fondements même de la société médiévale: le rapport aux origines, à l'autorité, au divin, le sentiment d'appartenance à une société, la relation au corps, le sens du couple, de la famille,... autant de questions qui rejaillissent dans notre monde de fin de millénaire, parti lui aussi à la recherche de ses pères. 


\section{NOTES}

1. Cette enquête, dont nous présentons ici les premiers résultats, a fait l'objet de notre mémoire de DEA sous la direction de Jacques Chiffoleau, Saint Joseph, une image de père à la fin du Moyen Âge, Université Lumière-Lyon 2, 1996. Nous poursuivons ce travail dans le cadre d'une thèse sur l'image de la paternité à la fin du Moyen-Age, autour de la mise en valeur de figures comme saint Joseph ou Dieu le Père.

2. Encore faut-il définir ce qu'est un lien «naturel»! Voir notamment J. Chiffoleau, «Contra naturam. Pour une approche casuistique et procédurale de la nature médiévale ", Micrologus, IV, 1996, pp. 265-312.

3. C'est outre-atlantique qu'il faut aller chercher des travaux plus complets sur l'époux de la Vierge. Au Canada, placé depuis le XVII ${ }^{e}$ siècle sous le patronage de saint Joseph, des études très poussées ont été stimulées par l'oratoire de Saint-Joseph à Montréal qui publie depuis 1953 des Cahiers de joséphologie. Ceux-ci sont bien entendu marqués par une position théologique et ecclésiologique, mais la qualité de leur érudition rend de grands services à l'historien. Sur le plan iconographique, il faut se reporter à la bibliogaphie de l'excellent ouvrage de R. Mellinkoff, Outcasts : Signs of Otherness in Northern European Art of the Late Middle Ages, Berkeley - Los Angeles, 1993, p. 79, note 146.

4.J. Huizinga, L'automne du Moyen Âge, rééd. Paris, 1989, p. 175.

5. L. Réau, Iconographie de l'art chrétien, Paris, 1958, t. III, vol. 2, p. 753.

6. J. Wirth, L'image médiévale, Paris, 1989, p. 308.

7. L. O. Vasvari, «Joseph on the Margin : the Mérode Tryptic and Medieval Spectacle ", Mediaevalia, vol. 18, 1995, pp. 163-189.

8. Jean Gerson, Oeuvres Complètes, éd. P. Glorieux, Paris, Tournai, New York, 1966, t. V, 232, p. 353. Sur l'utilisation de la formule d'Horace, fréquente à la fin du Moyen Âge, voir A. Chastel, «Le dictum horatii quidlibet audendi potestas et les artistes (XIII ${ }^{e}-\mathrm{XVI}^{\mathrm{e}}$ s.) », Fables, Formes, Figures, Paris, 1978, pp. 363-376.

9. Le développement de l'aspect iconographique de notre sujet déborderait le cadre de cet article. Signalons seulement que la figure de Joseph apparaît souvent en contrepoint d'un autre personnage : Dieu le Père dans la Nativité, Gaspar dans l'Adoration des Mages, etc...

10. Voir les travaux de Jean-Claude Schmitt et Jérôme Baschet, notamment J.-C. Schmitt, «La culture de l'imago », Annales Histoire, Sciences Sociales, janv. fév. 1996, n 1 , pp. 3-36.

11. Un des premiers exemples de ce type est la Nativité des Très Belles Heures du duc de Berry (Paris, BNF, nouv. acqu. lat. 3093, fol. $42^{\text {r }}$ ).

12. Par exemple le Ludus Coventriae (Cf. R. Woolf, The English Mystery Plays, Londres, 1972), et du côté français, les grandes Passions d'Eustache Mercadé et Arnoul Gréban (Cf. J. Subrenat, «Jésus et Joseph dans les Mystères du XV $\mathrm{XV}^{\mathrm{e}}$ siècle. Une curieuse relation Père-Fils ", Les relations de parenté dans le monde médiéval, Sénéfiance n²6, Aix-enProvence, 1989, pp. 560-562).

13. Jean Gerson, op. cit., t. VII, 230. Cf. M. Lieberman, «Lettre de Gerson au duc de Berry », Cahiers de Joséphologie, t. IX, 2, 1961, p. 236.

14. Bonne exhortation generale pour la feste de la Desponsacion Nostre Dame, Jean Gerson, op. cit., t. VII, 227. 
15. M. Lieberman, «Lettre de Gerson au duc de Berry », Cahiers de Joséphologie, t. IX, 2, 1961, pp. 202-205.

16. M. Lieberman, «Chronologie gersonienne. IV.Gerson poète », Romania, LXXVI, 1955, p. 327, situe la rédaction de ce texte entre le 26 septembre et le 23 novembre 1413, car il trouve une allusion à ces Considérations dans la lettre au duc de Berry.

17. Modo quodam scholastico plus quam declamatorio et accurato sermone, Jean Gerson, op. cit., t. V, p. 344.

18. Voir la chronologie établie dans M. Lieberman, «Chronologie gersonienne.

IV.Gerson poète », Romania, LXXVI, 1955, note 2 pp. 326-329.

19. Jean Gerson, op. cit., t. VII, 300, p. 67.

20. Cf. M. Lieberman, «Autour de l'iconographie gersonienne », Romania t. 84, 1963,

t. 85, 1964, t. 91, 1970.

21. Souligné par nous.

22. Cité par M. Lieberman, «Lettre de Gerson... », loc. cit., p. 238.

23. Reddatur Ecclesia unico viro vero et certo, Summo Pontifici sponso suo vice Christi. Jean Gerson, op. cit., t. V, 232, p. 362. Gerson ne développe pas davantage cette image, qui pourrait être ambigüe : elle donne à penser que Joseph est une figure du pape en tant qu'époux et gardien de la Vierge, symbole de l'Église, mais c'est le Christ qui est le véritable époux de l'Église. On retrouve donc ici la délicate position de Joseph, père et époux «à la place » d'un autre.

24. Jean Gerson, op. cit., t. VII, 300, p. 70.

25. Ibid., pp. 69-70.

26. Au-delà du débat politique, c'est aussi le principe de succession qui s'affirme ici. Cf. J. Krynen, «Le mort saisit le vif. Genèse médiévale du principe de l'instantanéité de la succession royale française », Journal des savants, 1984, pp. 187-221.

27. Jean Gerson, op. cit., t. V, 232, p. 347.

28. Puto tamen probabilius quod regnum Christi super Judaeam non fuit temporale sed spirituale sicut in aliis regionibus. Ibid.

29. BNF lat. $1485^{2}$. Cf. Ibid., pp. 42-44.

30. Ibid., p. 44.

31. Jean Gerson, op. cit., t. V, 248, p. 542. Cf. P. Glorieux, «Saint Joseph dans l'œuvre de Gerson », Cahiers de Joséphologie, t. XIX, 1979, p. 417.

32. Jean Gerson, Considérations sur saint Joseph, op. cit., t. VII, 300, pp. 73-74. Cf. aussi le sermon Jacob autem genuit, ibid., t. V, 232, p. 348.

33. Rappelons que l'expression de " père putatif » traditionnellement attribuée à Joseph provient de l'Evangile de Luc $(3,23)$ qui mentionne que Joseph était «à ce qu'on croyait (ut putabatur), fils de Joseph».

34. Quae igitur causa exstitit, ut eum qui pater non fuit, patrem esse memoraret? Qui simplici expositione contentus est, dicit : honoravit eum Spiritus sanctus patris vocabulo, quia nutrierat Salvatorem. Qui autem altius aliquid inquirit, potest dicere : quia generationis ordo a David usque ad Joseph deducitur, et ne videretur frustra Joseph nominari, qui pater non fuerat Salvatoris, ut generationis ordo haberet locum, pater appelatus est Domini. Homiliae in Lucam, PG 13, 1842-3.

35. Saint Augustin, De cons. evang., lib. 2, c. 1, n. 3, PL 34, 1071-72 ; De nupt. et concup., lib. 1, cap. 11, n. 12, PL 44, 421. Saint Ambroise, In Luc, lib. 3, cap. 3, v. 23, PL 15, 1589. Saint Ephrem de Syrie, «De supernaturali B.V. Partu, Sermo 148 », S.Ephraem Syri opera 
omnia, éd. Assemani, Rome, 1732-1746, t. 2, pp. 276-277. Cf. R. Gauthier, « La paternité de saint Joseph ", Cahiers de Joséphologie, t. V, 1957, p. 174 sq.

36. Cf. J. Mulliez, «La désignation du père », J. Delumeau et D. Roche (dir.), Histoire des pères et de la paternité, Paris, 1990, pp. 27-54.

37. Nec vir ergo matris, nec filii pater exstitit, quamvis certa et necessaria dispensatione utrumque ad tempus et appellatus sit, et putatus. Homilia 2 super Missus est, n. 15, PL 183, 69.

Cf. R. Gauthier, art.cit., p. 36.

38. Cela va dans le sens d'une spiritualisation du sacrement de mariage, et d'un contrôle plus strict de la sexualité.

39. Jean Gerson, op. cit., t. VII, p. 64.

40. Considérations sur saint Joseph, op. cit., t. VII, p. 76.

41. Ibid., p. 66.

42. Ibid., p. 64.

43. At vero quia corpus fuit ipsius Joseph jure matrimoniali quo fit mutua translatio corporum viri ad mulierem et e contra, videamus si cum intelligentiae sobrietate dicere fas nobis sit quod ex corpore et carne Joseph natus est Jesus Christus. Et hoc dici forsitan posset nisi piarum aurium timeretur offensio. Sermo Jacob autem genuit, op. cit., t. V, p. 357.

44. Fuit itaque Joseph pater Jesu reputatione; fuit pater curatione quia nutritius ; fuit tertio pater generatione, non quidem sua sed Mariae uxoris suae, cooperante Spiritu Sancto et quodammodo vices Joseph gerente, non virili semine sed mystico spiramine. Propterea Joseph pater non quidem naturalis sed legalis pueri Jesu dici potest cui Spiritus Sanctus suscitavit semen carnali valentius. op. cit., t. V, p. 357.

45. Maria de qua natus est Jesus sicut fuit in utero sanctificata priusquam nasceretur, ita de Joseph virginali viro suo pia probabilitate credi potest quamvis forte non omnino similiter. Potest forsan haec dissimilitudo notari in hoc quod Joseph post originale contractum sanctificatus est in utero baptismo flaminis sicut Joannes Baptista et aliorum plurimi. Ibid., p. 349.

46. Jean Gerson, op. cit., t. VII, 300, p. 67.

47. La primauté de Jean Baptiste, seul saint dont l'Église célèbre la naissance, est affirmée par exemple par saint Cyrille d'Alexandrie (Thesaurus, PG, 75, 157), saint Jean Chrysostome (In Matth., homil. 37, n. 2, PG, 102, 421), saint Augustin (Contra adversarium legis et prophetarum, 1. II, c. V, n .20, PL, 42, 650 ; Sermon 290, 2, PL 38, 1313 ; Sermon 292, 2, PL 38, 1321a). Cf. A. Michel, "Joseph (saint) », Dictionnaire de Théologie Catholique, VIII, 1925, 1514, et D. Buzy, Saint Jean-Baptiste, Paris, 1922.

48. Quamvis in utero usum liberi arbitrii non habuerint. Somme théologique, $3^{\circ}$ p., qu. 27, a. 6. Cf. D. Buzy, op. cit., p. 87.

49. Joannes et Jeremias, quamvis sanctificati in uteris matrum, traxerunt tamen originale peccatum. D. Buzy, op. cit., p. 80.

50. Cette place mériterait d'ailleurs une étude plus approfondie. Cf. l'ouvrage vieilli et trop général d'Alexandre Masseron, Saint Jean Baptiste dans l'art, Paris, 1957.

51. Jean Gerson, op. cit., t. VII, 300, p. 67.

52. Cité par M. Lieberman, «Pierre d'Ailly, Jean Gerson et saint Joseph », Cahiers de Joséphologie, t. XV, 1967, p. 48.

53. Cf. J.J. Lemire, "La pensée théologique de Bernardin de Bustis sur saint Joseph », Cahiers de Joséphologie, t. XIX, 1971, pp. 481-496. 
54. Par exemple par Suarez, De mysteriis vitae Christi, disp. VIII, sect. 2, n. 6-8, puis Benoît XIV, De servorum Dei beatificatione et beatum canonizatione, 1. IV, part. II, c. XX, a. 31, Padoue, 1743, p. 135.

55. Le texte du décret ne nous est pas parvenu, mais la fête de saint Joseph est introduite dans le bréviaire romain publié à Venise en 1479. Cf. J. Dusserre, «Les origines de la dévotion à saint Joseph », Cahiers de Joséphologie, t. II, 1954, p. 26. 56. Par exemple deux sermons de Nicolo Pieri de Sienne, élu provincial de Lombardie en 1339, édités par A. Barriston, «Deux sermons du fr. Nicolo Pieri de Sienne ", Cahiers de Joséphologie, t. VII, 1959. Chez les Franciscains, on trouve un long chapitre consacré à Joseph dans l'Arbor vitae crucifixae Jesu d'Ubertin de Casale, en 1305, puis dans le De vita Beatae Mariae Virginis de Barthélémy de Pise. Ces deux textes sont repris dans un grand sermon latin de Bernardin de Sienne (Opera omnia, Lyon, 1650, t. IV, p. 250). Cf. J. Dusserre, loc. cit., Cahiers de Joséphologie, t. I, 1953, p. 186, 191, t. II, 1954, p. 18.

57. Jean Gerson, op. cit., t. V, p. 349.

58. Cf. I. N. Maegawa, «La doctrine de Jean Gerson sur saint Joseph », Cahiers de Joséphologie, 1960, pp. 271-277, et P. Lucot, Saint Joseph, étude historique sur son culte, Paris, 1875, pp. 123-164.

59. M. Lieberman, «Les sources joséphologiques de Gerson et l'office des Carmes », Cahiers de Joséphologie, t. X, 1962.

60. Tours, Bm, ms. 156 et 148, complétés par quatre autres manuscrits, notamment le ms. 352 de la Bibliothèque Mazarine. Cf. Lieberman, loc. cit., pp. 190-192.

61. Nam in ventre matris iste / Nazareus tibi, Christe / Consecratur et notatur. Ibid., pp. 219-220.

62. Cf. M. Lieberman, «Lettre de Gerson... », loc. cit., pp. 204-205, et Id. « Pierre Pocquet : Dictamen de Laudibus Beati Joseph », Cahiers de Joséphologie, t. XII, 1964.

63. Edité par R. Gauthier, Cahiers de Joséphologie, t. I, 1953. Cf. M. Lieberman, «Pierre d'Ailly, Jean Gerson et saint Joseph », Cahiers de Joséphologie, t. XV, 1967.

64. P. M. Branchesi, «Una chiesa bolognese dedicata a S. Giuseppe dal sec. XII », Cahiers de Joséphologie, t. XIX, 1971, pp. 576-590.

65. B. Burkey, «The Feast of Saint Joseph : A Franciscan Bequest », Cahiers de Joséphologie, t. XIX, 1971, p. 648.

66. On peut citer une exception remarquable, celle d'un mystique allemand du XIII siècle, le bienheureux Hermann - Joseph de Cologne, prémontré à Steinfeld, qui décida lui-même de changer son nom en celui de Joseph et de vivre sur le modèle de l'époux de la Vierge. Cf. J. Dusserre, «Les origines... ", loc. cit., p. 173. Ces données seraient à compléter avec les récents travaux d'anthroponymie du groupe de la Sorbonne.

67. Cf. supra, note 56 .

68. Cf. V.A. Kolve, A Play Called «Corpus Christi», Stanford, 1966, p. 247.

69. Y. Pelicier, «Avant-propos » dans H. Tellenbach (dir.), Das Vaterbild in Mythes und Geschichte, Stuttgart, Berlin, Köln, Mainz, 1976, éd. française L'image du père dans le mythe et l'histoire, Paris, 1983, pp. 7, 11, 12. 


\section{AUTEUR}

\section{PAUL PAYAN}

9 ch. des amandiers, 30400 Villeneuve-lez-Avignon 